\title{
Mediated Interpersonal Communication: A New Way of Social Interaction in the Digital Age
}

\author{
Yuli Candrasari ${ }^{1}$ \\ ${ }^{1}$ Universitas Pembangunan Nasional "Veteran" Jawa Timur Surabaya, Indonesia \\ Corresponding Author's Email : yuli_candrasari.ilkom@upnjatim.ac.id
}

\begin{abstract}
Although previously intended to seek information, the Internet becomes a means to communicate and social interaction. Thus, it has since proven that social media mediates interpersonal communication, connecting people that never met in face-to-face situations. Social media continuously shaped and shifted the way netizens interact amongst themselves This paper seeks how the netizens interact with each other and how this interactivity takes place within social media realms. Therefore, Social Construction theory and Social Information theory will be used to answer these questions. Using this method of research netnography was done on Bening Society community Facebook account. The informants in this study were drawn from members of the active Bening Society community. Netnography is utilized as a method and the Bening society on Facebook which garnered thousands of members is used as a case study. Hence, the informants are those who are active in participating in this online society. The results showed that social media, in this case, Facebook is able to provide comfort for users, especially in managing social interaction with friends. The process of interpersonal communication that occurs through Facebook resembles those in face-to-face interpersonal communication.
\end{abstract}

Keywords: social media, CMC, interpersonal communication, Facebook, theory of social information processing

\section{INTRODUCTION}

The presence of the internet that is accessible to all people throughout the world has accustomed people to enjoy the quality, range, and choice of entertainment and information content through new forms of communication. This new form of communication provides many opportunities with the mass media that have been known so far. This new media is very open, cheap and easy to access, besides this new media provides various types of information to its users.

In its development, at this time new media emerged as the mainstream media for information exchange and social interaction throughout the world. Many people have used the new media not only for information search but also for communication. Not infrequently among users also become providers of information even today most users contribute to content through blogs, wikis, and the most recent podcasts and videos [1]. This has become one of the attractions of this new media compared to the mainstream media. Therefore internet users from year to year always increase rapidly. In Indonesia in 2018 internet users have reached 171.71 million (APJII 2019). 
This increased internet users occur in Indonesia very rapidly. The average penetration of internet use in urban areas is still 30-35 percent in 2010, and has since increased to 40-50 percent in 2011 based of MarkPlus Insight.. MarkPlus Insight asserts that the number of Internet users in Indonesia in 2012 has reached 61 million people, an increase of 6 million from the year 2011 amounted to 55 million. The most visited sites are social media, especially social networking sites. The Facebook site ranked first as the much-visited sites beat Google search engine sites. A study says that $62 \%$ of children access the internet for social media sites. While as much as $43 \%$ to access to online games and 23\% to do "dating" (Norton Online Living Report, 2009).[2]

The popularity of social networking sites,becoms the new way of communication among netizens. A study by Lenhart (2009)[3] on "How Youth Communicate Every Day" shows that 12-17 year olds as many as 51\% communicate via the Internet by accessing social networking sites. Only $29 \%$ of teenagers communicate with their friend's face-to-face communication (face-to-face communication). This shows how the virtual world (through social networking sites) has been able to replace the form of communication in the social life of its users. Research conducted by Pelling, L, Emma[4] (2009) against internet users aged 17-24 years shows that in a day they visit social networking sites four times and sometimes more. The high usage of social networking sitesamong adolescents due to adolescence in the development of psychological teenagers always want to group or make friends as much as possible. In addition to the basic nature of human beings as social beings who want to always interact with their social groups..

Through social media everyone can manage their daily activities such as making appointments, finding information, shopping, making purchases without having to meet physically. People today are becoming highly dependent on the internet, where the internet is not only a medium for getting information and communicating but also as a means of socializing.

One form of social media is Facebook, a social networking site that can connect users with anyone in the virtual world and can connect with old friends. The Facebook site ranked first as the much-visited sites beat google search engine sites. A study mentions that 62 percent of Internet users are 18-34 years old in accessing the internet for social media sites. (Norton Online Living Report, 2009)[2].

Through Facebook, individuals can interact and communicate with their network friends. Facebook helps its users build social interaction and interpersonal communication (mediated) amongst their users like the real world although they are separated physically.

Such phenomenon is interesting because but the process of interpersonal communication can run well without face-to-face interaction. Some netizens even successfully manage friendship amongst them, solely through online communication. This research seeks to answer how the netizens of Facebook (in The Bening society) manage their social interaction solely through online.

\section{THEORETICAL FRAMEWORK}

One study conducted by Ronald and Caroline, in Lievrouw Leah A and Sonia (2002: 102) concluded that for the optimistic group in viewing the internet presence that the internet is seen as a medium to enhance social interaction[5] Several studies on CMC 
(Computer-Mediated Communication) have shown that CMC is not necessarily less personal than face to face communication. Research conducted by Ando and Sakamoto (2008) states that having a cyber friend can reduce feelings of loneliness and social anxiety in individuals and increase closeness and intimacy with friends who have been known before. Basically communication through social media can make individuals easier to develop networking. As a result of Williams, N., Nakeshia (2016)[6] which states that social networking sites provide a means for individuals to present themselves by first constructing their identity, thoughts and photos of themselves to be distributed to others. While research conducted by Park and Roberts (1998) in Ronald E. Rice [7](2002) states that although the frequency and duration of online are lesser, the interactions and communications that are interwoven in online in enhancing individual relationships are more intimate and can be well established. Likewise the results of research conducted by Pollet, Roberts, GB, and Dunbar, MIR (2011: 255) which revealed that individuals who use instant messaging and social networking sites have greater group support, good group sympathy, and more friendship many than those who don't use social media[8]. Some research on the use of social media states that currently, individuals when accessing the internet spend a lot of time on social networking sites (Yuan, 2015: 15). In addition to the easy and inexpensive factors that cause individuals to spend time on social networking sites, there are social factors that also cause many social networking sites to be accessed. Namely the characteristics of individuals as social beings who always want to establish social relationships with other individuals.[9]

\section{Theory of Social Information Processing}

Although interpersonal communication mediated in cyberspace can not fully engaged nonverbal symbols in face-to-face interpersonal communication, it is very meaningful for the sustainability of communication. As stated in the cues-filtered-out perspective that nonverbal cues are a necessary cue to form positive impressions and warm relationships with others online. Therefore, Information Processing Theory argues that CMC which is only text based does not mean to have limitations on access to verbal cues and should not be considered to be a weakness inherent in the CMC that makes the CMC less useful in building social relationships.

The Social Information Processing Theory (SIP) is expressed by Joseph Walther (1992) who focuses on how the individual in the virtual world "forms the impression through the textual information it conveys" (Walther, 2011: 10)[10]. This theory also reveals that social information can arise slowly despite the lack of non-verbal symbols and on asynchronous media that have many channels on line. This means that with sufficient time, the result of establishing relationships in cyberspace may be the same as when individuals build face-to-face communication relationships. This Social Information Processing Theory explains how CMC has less nonverbal cues but is able to encourage the creation of personal relationships. Based on that perspective, Walther stated that the actual communication through the computer can take place effectively as face-to-face communication if it can optimize the two aspects of the support of verbal cues and temporal cues.

Similar to the theory of social penetration and the theory of uncertainty reduction, the Social Information Processing Theory also explains the development of relationships. According to Walther in the theory that there are two factors that determine the 
relationship formation process, namely the verbal and temporal cues. This theory uses these two factors as parameters in which communication and technology can combine to produce impersonal, interpersonal, or hyperpersonal relationships.

Walther stated that actually, communication through a computer can take place as effectively as face-to-face communication if it can optimize two supporting aspects, namely verbal cues, and temporal cues.

a. Verbal cues

Information Processing Theory does not argue that CMC has limitations on nonverbal cues. This theory states that this message typed on computer media is equivalent to verbal cues on face-to-face media. However, this theory refutes claims that the shortcomings would make $\mathrm{CMC}$ useless in forming impressions and building relationships. Information processing theory believes that individuals can adapt to the limitations that exist in $\mathrm{CMC}$ by using verbal cues to the maximum to convey social information and relational messages.

\section{b. Temporal Cues}

The Social Information Processing Theory also states that the temporal or the length of time a communicator has to exchange messages is a central influence on the type of relationship formed. Logically, if the communicator only has one channel to use for communication through CMC then it will take longer than face-to-face media to achieve the same goal (building relationships). Walther in Ramirez, A. (2009: 898) argues that when the communicator is given a limited time to exchange messages then what happens is an impersonal relationship.[11] This is because there is no closeness or close relationship between the individuals involved. Conversely, when individuals are allowed to exchange messages without temporal constraints, Walther predicts that mediated interpersonal relationships are built showing the level of development of results will be comparable to those developed by face-to-face communication.

In certain circumstances, and the reduction of obstacles can lead to relationships that are built through interpersonal mediation mediated levels of familiarity achieved can exceed relationships built through face-to-face communication. Relationships like these that Walther calls hyperpersonal relationships.

\section{METHODOLOGY}

This research was conducted qualitatively with the Netnography method. Kozinets in the book Netnography defines netnography as "an ethnographic form adapted for the computer-mediated social world" (2010: 10)[12]. In short, netnography is a method for studying cybernetics space (cyberspace). Netnography is appropriate to assess both online communities and cultures in online communities where in their cyberspace interact socially.

\section{Determination of Data and Data Sources}

In this netnography research, researchers are required to engage and interact with each other. This means the researchers must be involved in the virtual reality. This study focuses on social networking sites, like Facebook.

Choosing informants requires thoroughness and patience because not all Facebook accounts have a high traffic. "Traffic" is one of the requirements in selecting 
informants in netnografi. Kozinets (2002: 63) writes there are several criteria for selecting online informants (1) focusing on questions or research topics;

(2) based on a high "traffic" post; (3) based on the number of different messages (comments); (4) more detail post (5) interaction between member or not high member as required in research[13].

Based on these criteria the researcher Facebook account that not only has a large number of followers but also the frequency of posts that occurs in the Facebook account. The "Bening Society" has been chosen in this study because this community this community has been around since 2012 with large members. This community is a community of women of reproductive age. They originally joined because of the similarity "profession" that is as an online entreperneurs. Uniquely this community is a community that although open but does not have male members. To date, the community has 5050 members and is active.

Eight members of the Bening society has been interviewed for the purpose of this study. Data gathering methods are participant observation,indepth interview and literature study. Data analysis was done by categorizing data for analysis.

\section{RESULTS AND DISCUSSION}

Facebook is one form of social media has a feature that is quite complete in supporting users to communicate and interact with other users. Facebook has features like just Instant Messaging ie "Inbox / Messenger" so that personal messages can be delivered even with these features users can chat directly with other facebook users. Facebook also provides a feature "wall", if users want to convey messages addressed to social networking friends.

The results showed that all informants are women who have a considerable ability of access to social media especially Facebook. Research data show that informants never sign out from Facebook. This means that every time they can access Facebook. Some research on the use of social media states that currently, individuals when accessing the internet spend much of their time on social networking sites (Tsitsika, A., et.al, 2008)[14]. In addition to the easy and inexpensive factors that cause individuals to spend time on social networking sites, there are social factors that also caused many social networking sites accessible. That is the characteristics of individuals as social beings who always want to establish social relationships with other individuals. With the completeness of features, Facebook enables the human needs to interact with each other as social creatures.

The results of this study reveal how much Facebook as a social media is able to facilitate users in conducting interpersonal communication although separated by distance and time. The author further calls this form of communication through the medium (social) as Mediated Interpersonal Communication. Walther (2011) states that in social relations through social media, interpersonal communication occurs mediated among individual users.[10]

This research data also shows how the informant as an active Facebook user can perform interpersonal communication (mediated) with his friends as face-to-face interpersonal communication. In fact, interpersonal communication mediated through Facebook is not much different roles and functions with interpersonal communication face to face. For informants using facebook as a medium to interact and communicate 
with his friends. Therefore this research data shown the existence of communication activities on informants when using facebook, such as 1) make communication and interaction with friends facebook; and 2) building social relationships in social media.

\section{a. Communicate and Interact with Facebook Friends}

The results of this study show that through Facebook informants get many friends and can reconnect relationship with old friends. Facebook as a personal media has made it easier for its users to engage in mediated interpersonal communication.

The features provided by Facebook make it possible for its users to be able to easily connect with friends also get new friends. The phenomenon shows that in fact Facebook as a social media has facilitated its users for the occurrence of interpersonal communication as inter-personal communication in face to face. As one of the functions of interpersonal communication is to build and maintain relationships between individual (Suranto, 2011: 7) [15]. Facebook has facilitated the informant in building and maintaining relationships with his friends, not only his old friends but also new friends. Facebook as a personal media has made it easier for its users to engage in mediated interpersonal communication.

The research data also revealed that Facebook has made informants feel strong bonds of friendship especially when they enter a community. It can be seen how the informant feels a new "family" in the Bening Society community, although they have basically never met physically and did not know each other before. The social bond is well established in the Bening community and there is an emotional closeness among its members. As informant says about how he feels he's getting a good friend even though they do not know each other before:

"You can meet another brands' lovers and enthusiasts. When you connect with them deeply, it feels closer than siblings"

In addition, the researchers also get some statements or expressions from Bening Society residents who posted on the Facebook wall page Bening Society as well as on the comments page. The post revealed the closeness among Bening residents using the words "mbak say" which is a form of calling the citizens Bening to other Bening residents. The call "mbak say" is an extension of the phrase "mbak sayang". The form of calling in the culture of the Javanese community, in particular, is to point to a form of homage to others and usually, this call is directed to a brother who has a kinship relationship. Therefore the call "mbak sayang" can be interpreted as a form of closeness and respect for other individuals. The use of the call indicates that there are close emotional ties among Bening members they have never known and met physically.

As Zheng, Burror, Drew (2010: 56) [16] stated that individual relationships in cyberspace are strong when they have emotional closeness, intimacy, mutual trust, sharing, and self-disclosure. Things are very different in face to face communication. In face-to-face communication at the beginning of the meeting each party involved in it will keep each other distant and looking for information first including looking for similarities that exist between them so that the communication process can run well. This fact reinforces the researcher's assumption that social media (Facebook) is easier to use for individuals in building social relationships. Especially for individuals who have difficulty socializing in the offline world then when they use social media can feel the ease of building social relationships.

In Facebook communication behavior is when netizen can communicate with each other directly with his friends and they can also express individual support to others by giving 
comments on posting his friends. Doing posts on the page Facebook wall. includes send a message to the inbox Facebook friends and do "chat" via messenger. Researchers observe on the personal account of each informant that there are efforts of informants to maintain communication and interaction that has been established that by always communicating actively with his Facebook friends. One of the findings of the data shows that the informants in this study are active to provide comments or simply put a "Like" sign on the posts of his friends on the Bening Society facebook page. No wonder if the post informant on Facebook account Bening often "flood" comments and signs "Like". Similarly, when researchers observe the personal account of informants, each time the post informant always get a response both in the form of and the sign "Like". Providing a "Like" sign or emoticon symbol is an attempt by netizens to keep in touch and maintain the social relationships they have intertwined.

\section{b. Building Social Connections on Facebook}

As has been described in the future that the current existence of the internet not only serves as a means of seeking information as the motive of the birth of the internet in the past. The birth of social networking sites like Facebook has made the function evolve. The Internet is not only as a means of seeking information but nowadays internet function is more dominant to build communication and social relation with cyberspace community.

Research data shows that Facebook facilitates users (informants) in building communication and social relationships through several stages, the first stage of receiving friendship, the second stage is self disclosure among Facebook users and the third stage is mutual support.

At this stage of accepting this friendship, the informant will immediately receive when the party who asks for friendship is his old friend or a friend he already knows. But to foreigners (unknown informants) then informants will be careful to accept friend requests. Informants will make observations and assessments on three things: see the profile of self that is listed on personal information, that is by looking at the profile picture, name and identity self contained in the personal information. Furthermore, informants see postings that the owner of the facebook account on his facebook wall page. The posting will be considered netizens in assessing the "character" of the stranger. The last informant will see the mutual friends that the stranger possesses. If there is an equation friends who are owned by their social networking friends then it becomes a netizen's consideration to accept friendships from strangers. The three categories will determine whether the netizen receives a request for acceptance from a stranger or refuses the stranger's friend request.

At the stage of self-disclosure revealed in this research that the informant was easier to express themselves or open to friends social network even though they just know each other. They are also accustomed to discussing many things, ranging from the problem of Muslim fashion products, femininity, family, social, religious, even up to political issues. The phenomenon shows that Facebook is able to make its users build communication and good relationship so that among them feel comfortable to more open. This is because facebook is able to build an emotional relationship with its users. As the following informant 2 discloses: 
"Yeah sist.. We all started to know in the group. To be a good friend. In the beginning, she bought my bag... Finally we often chat, chatting often, until Icome to her house in Jakarta .. Buy a round-trip ticket, invited the streets around Jakarta".

The same phrase is also expressed by the following fifth informant: "... wahhhh I am so happy sist, "honey b" first meet like the family already. Until now we are familiar with each other's families though not every week".

Through Facebook citizens Bening Society meet and know each other. The communication process that intertwine leads to the emotional closeness among them. As Zheng et al (2010: 56) [16] pointed out that an online relationship is said to be of good quality if among its members can share messages openly, there is a message that shows emotional closeness, openness, mutual trust and they can communicate about many things .

The existence of such openness in addition to the proximity among Bening residents but also because all citizens of Bening Society are women. In the virtual world of women tend to be easier to do self disclosure than men. The results of this study also reinforce the statement especially when they are involved in a community whose members are all women so that the informant feels safe and comfortable to tell anything.

The absence of "distance" between netizens in communicating through Facebook makes netizens easy to feel comfortable in communicating. As in face-to-face communication, when individuals meet strangers there will be "distance" between them so that the communication process that runs feels "rigid" and limited. On the Bening Society community Facebook page, the communication process happens intensely and continuously even though members of the community are actually reunited through Facebook. Forms of support were often being provided by Bening residents when there is one Bening residents who are experiencing misfortunesor when among Bening people want to get information about good online sellers in the sense of being trustworthy or simply information about education, schooling, parenting, hobbies and more.

This form of support can be a concern given by netizens to other netizens. One of these concerns is that routine informers read status updates not only on the Bening Society community Facebook page but also on the personal Facebook page owned by every member of the Bening Society. To show support to friends, Facebook especially incorporated in Bening Society informant actively comment on the status of his friend or just a sign "Like" on the status of his Facebook friends. Thus the relationship of friendship in cyberspace will be maintained. As human social character that there is a symbiotic mutualism when individuals want their social relationships well preserved.

The form of support here is not only emotional support but also information support. Emotional support is already visible on how among Bening members give each other attention and have a good understanding. At this stage informational support is also obtained by informants who are part of the citizens of the Bening Society community. These two forms of support make bonding among Bening Society's citizens stronger. Nancy K Baim [17] (2010: 75) also wrote that it is said to be a strong online community if there is a sharing of resources and support among members of the community. Social support in an online community will provide many benefits to community members. 
Community members will find it easier to adjust, better solve problems, and improve performance. Conditions like these are also perceived by informants as citizens (members) of the Bening Society community. The rapid process of the new Bening members in adapting to the "environment" of the Bening Society community includes obeying the rules of the community. This is because those who join the community because they have similar interests.

\section{Interpersonal Communication Mediated as a New Communication Form}

In the past it was never thought that interpersonal communication could be done through the media. Nowadays, when the internet with the speed of innovation gave birth to social media platform then interpersonal communication can be done with media intermediaries. This is what the author calls a new form of interpersonal communication, namely mediated interpersonal communication.

The presence of social media increasingly confirms that CMC benefits its users to establish social interaction and communication. One of the characteristics of CMC is interactivity, namely the existence of two-way communication. Characteristics it possesses is found on social media as one form of CMC. Therefore, by using social media, netizens can establish interpersonal communication in cyber world with ease.

As the results of the study and supported by several other studies that say that most netizens use the Internet more to open social networking sites than to access the search engine site. Even this research also shows that social media (Facebook) is never separated from daily activities.

This Social Information Processing Theory is one of the theories that specifically examines interpersonal communication in CMC. Social Information Processing Theory rejects the notion that the absence of nonverbal cues in interpersonal communication through the medium (internet) will reduce the quality of communication, namely the formation of closeness among the individuals involved in it. The Social Information Processing Theory states that the absence of nonverbal cues does not reduce the "warmth" of the participants of communication but rather with the media's lack of will enable individuals to adapt to build intimate social relationships.

This is what happened to the informants in this study. Through Facebook they "meet" and introduce each other to establish communication and interaction. There is not much time netizens need to start communicating and even accept friendships from other people he or she does not know at all. These facts show that even if they do not meet physically, the disappearance of nonverbal cues such as performance, gesture, intonation of voice, does not make netizens find it difficult to build interpersonal (mediated) communication. These conditions indicate that netizens are able to adapt to the "limitations" that exist in social media as one form of the CMC.

This phenomenon was initiated by Walther (1996)[10] in Social Information Processing Theory. This theory examines how text-based CMCs - no nonverbal cues can be used to perform interpersonal communication just like face-to-face communication. Even Koh (2002) as quoted by Serena Leow, B.A. (2009: 25) states that online users need not worry about their physical appearance in cyberspace because it can be regulated (engineered). Koh instead emphasizes the "utterances" that are typed in text form for online users to build impressions for other users. Online users have more time to design textual content and present themselves in the online social world.[18] 
In face-to-face communication, individuals when wanting to talk or want to express their feelings must first meet physically because the message is limited in terms of coverage. Face-to-face communication requires first recognition among individuals involved in communication to gain closeness and support from others.

In face-to-face communication, to build a relationship hence presence of physical and non verbal cues are required such as facial expressions, body movements, appearance, accent or speech and intonation of sound as a factor supporting the establishment of a relationship. Both factors will be an assessment and consideration for the individual whether to continue communication or not (Baym K Nancy, 2015).[19]

In mediated interpersonal communication, in establishing relationships the individual does not assess nonverbal cues - such as face-to-face communication - but judgments are made through the social information he gets in CMC. As the results, this study reveals that informants building social relationships simply by viewing and performing a simple assessment of personal information through Facebook wall pages. Personal information obtained was not detail because not all information about the individual self-owner of facebook account written openly and "honest". Therefore in order to assess someone then the informant assess the postings on the page facebook wall of someone. Netizens build social relationships through two processes. This is in keeping with Walther's Theory of Information Processing, that the individuals build and develop relationships based on the social information they gets. When the individual feels impressed and can accept the self-construct that other individuals have built then the relationship will usually can grow into a closer relationship.

The results of this study also show illustrate that social media (facebook) is easier for users to build closer and closer relationships. This means that the relationship between members of the "Bening Society" community is quite strong even though in the beginning they did not know each other and the closeness was built only based on mutual interest (in this case the similarity of interest in the hijab) towards something without a clear and open self-presentation Facebook has made the relationship between users is quite close. As written by Miller M.S, Miller L.,

Kenneth and Alison C (2010: 56) that individual relationships in cyberspace are said to be strong when there is emotional closeness, intimacy, mutual trust, mutual sharing and self-disclosure (Self-Disclosure).[20] Very different things in face-to-face communication. In face-to-face communication at the beginning of the meeting each party involved in it will keep their distance and find information first, including looking for similarities that exist between them so that the communication process can go well. Also in face-to-face communication, communication will continue after the performance appraisal. Often the mistake of physically assessing a template will hamper the communication process and the individual's reluctance to build relationships.

In the online world, such things are not obtainable. In CMC, individuals may be unaware of what their interlocutors are like and how they are physically shaped. On social networking sites, individuals can easily build relationships and communicate without being "burdened" by judgments about themselves. It may be that new individuals "get to know the other person". with details of the interlocutor after a personal relationship has been built properly In the absence of physical presence, individuals feel more easily and freely in communicating with other individuals without having to think about their appearance (such as what) to be accepted by others properly. 
This fact reinforces the researchers' assumption that social media (Facebook) is easier to use for individuals in building social relationships. Especially for individuals who have difficulty socializing in the offline world so when they use social media can feel the ease in building social relationships. Some previous research results have written that social networking sites (including Facebook) help individuals in building social relationships. That social networking can make it easier for individuals to make new friends and even make it easier for netizens to become more familiar with their new friends even though they have never met. (Ellison, et, al, 2007).[21] This is in line with the Hyperpersonal perspective which reveals that individuals become more easily acquainted with others through internet-mediated interpersonal communication compared to face-to-face interpersonal communication. This condition is one of the factors driving netizens to use social networking sites. Through social networking sites, netizens will not feel alone. As the results of research conducted by Matsuba as quoted by Marie Allan (2014: 15) that when individuals do a "temporary stopping" to use facebook for 48 hours then what happens Facebook users feel lonely because no friends are invited to communicate.

This is because Facebook users feel more comfortable when they can build relationships through online communication. Facebook has succeeded in becoming a medium that facilitates social interaction among its users. This is because Facebook as a social networking site can provide emotional, motivational, informative support, and can provide advice among its users. CMC can make interpersonal relationships that are temporary become more friendly, intimate and can be established over a long period. Especially the netizens in the online community, because one of the benefits that individuals get when entering the online community is getting social support. Social support can be in the form of opportunities to build and maintain social relationships with other netizens as well as opportunities to share support emotionally (Iriberry \& Leroy, 2009: 118).[22] In addition, the emotional closeness felt by Bening Society residents is inseparable from the stereotype of women, that women are more sensitive in social relations, more attentive to the needs of others, more expressive in expressing their feelings, warmer, and more understanding, and more focused on harmony and tend to avoid conflict more. Conversely, men will be more dominant, more confident, independent, and prefer to hide their feelings (Fischer, A., 2011: 56).[23]

\section{BIODATA}

Yuli Candrasari, Aulia Rahmawati, and Catur Suratnoaji are a senior lectures at UPNV University, Indonesian.

\section{REFERENCES}

[1] D. N. N, "New Media and New Culture: The Dependency of Indian Online Community," IOSR J. Humanit. Soc. Sci., vol. 19, no. 10, pp. 42-49, 2014.

[2] Symantec Corporation, "Norton online living report," pp. 1-22, 2009.

[3] M. Madden, A. Lenhart, M. Duggan, S. Cortesi, and U. Gasser, "Teens and Technology 2013," Washington, DC Pew Res. Center's Internet Am. Life Proj. 2013, pp. 1-19, 2013.

[4] E. L. Pelling, B. B. Sc, K. M. White, and D. Ph, "The Theory of Planned Behavior Applied to Young People's Use of Social Networking Web Sites,” vol. 12, no. 6, 2009. 
[5] L. A. Lievrouw and S. Livingstone, "Handbook of New Media: Social Shaping and Consequences of ICTs," pp. 62-76, 2002.

[6] C. W. Lewis and J. H. Lim, "DIGITAL MIRROR : AN EXAMINATION OF SOCIAL MEDIA' S INFLUENCE ON LATE ADOLESCENT BLACK FEMALES' GLOBAL AND

ACADEMIC SELF - CONCEPT by Nakeshia N . Williams A dissertation submitted to the faculty of the University of North Carolina at Charlotte in partia," 2016.

[7] R. E. Rice, "New media/Internet research topics of the Association of Internet Researchers," Inf. Soc., vol. 21, no. 4, pp. 285-299, 2005.

[8] T. V. Pollet, S. G. B. Roberts, and R. I. M. Dunbar, "Use of Social Network Sites and Instant Messaging Does Not Lead to Increased Offline Social Network

Size, or to Emotionally Closer Relationships with Offline Network Members," Cyberpsychology, Behav. Soc. Netw., vol. 14, no. 4, pp. 253-258, 2011.

[9] W. C. Yuan, "The Influences of Dual Social Network Site Use and Social Capital Development on Sociocultural Adaptation," 2015.

[10] J. B. Walther, Theories of computer-mediated communication and interpersonal relations. 2011.

[11] A. Ramirez, "Social Information Processing Theory," Encyclopedia of Communication Theory. Sage Publishing.

[12] G. Bowler and G. M. Bowler Jr, "Netnography: A method specifically designed to study cultures and communities online," Qual. Rep., vol. 15, no.

5, pp. 1270-1275, 2010.

[13] R. V. Kozinets, "The Field Behind the Screen: Using Netnography for Marketing Research in Online Communities," J. Mark. Res., vol. 39, no. 1, pp. 61-72, 2002.

[14] "Internet_use_and_misuse_a_mul.pdf." .

[15] A. . Suranto, Komunikasi Interpersonal. Yogyakarta: Graha Ilmu, 2011.

[16] R. Zheng, J. J. Burrow-Sanchez, and C. J. Drew, Adolescent online social communication and behavior: relationship formation on the Internet. 2010.

[17] N. K. Baym, The Blackwell Handbook of Internet Studies. .

[18] B. A. Serena Leow, "YOU DON'T KNOW ME BUT CAN I BE YOUR FRIEND? ACCEPTING STRANGERS AS FRIENDS IN FACEBOOK," 2009.

[19] N. K. Baym, Personal Connections Age. Wiley \& Sons, 2015.

[20] M. S. Miller, M. L. Kenneth, and A. Christine, "Connected at any Cost: Adolescent Developmental Needs and Online Relationship formation," in

Adolescent Online Social Communication and Behavior: Relationship Formation on the Internet, R. Z. Zheng, J. J. Burrow-Sanchez, and C. J. Drew, Eds. Information Science Reference, 2010, pp. 50-67.

[21] C. S. and C. L. Ellison, N., “"The Benefits of Facebook 'Friends'. Social Capital and College Students' Use of Online Social Network Sites.," J. Comput., 2007.

[22] A. Iriberri and G. Leroy, "A life-cycle perspective on online community success," ACM Comput. Surv., vol. 41, no. 2, 2009.

[23] Fischer A, "Gendered Social Interaction in Face-to-Face and Computer-

Mediated Communication," in Face-to-Face Communication over the Internet: Emotions in a Web of Culture, Language and Technology., Cambridge University, 2011 